\section{DNA delivery into anterior neural tube of zebrafish embryos by electroporation}

\author{
Cathleen Teh, Shang Wei Chong, and Vladimir Korzh \\ Institute of Molecular and Cell Biology, Singapore
}

BioTechniques 35:950-954 (November 2003)

The zebrafish is widely used for functional studies of vertebrate genes. It is accessible to manipulations during all stages of embryogenesis because the embryo develops externally and is optically transparent. However, functional studies conducted on the zebrafish have been generally limited to the earliest phase of activity of the gene of interest, which is a limitation in studies of genes that are expressed at various stages of embryonic development. It is therefore necessary to develop methods that allow for the modulation of gene activity during later stages of zebrafish development while leaving earlier functions intact. We have successfully electroporated the green fluorescent protein $(G F P)$ reporter gene into the neural tube of the zebrafish embryo in a unidirectional or bilateral manner. This approach can be used for the functional analysis of the late role of developmental genes in the neural tube of zebrafish embryo and larvae.

\section{INTRODUCTION}

Following the completion of genome sequencing projects for human and several other model organisms, efforts were under way to assign a function to specific genes. To understand the function of genes, their overexpression or inhibition is widely used. The zebrafish is a model system that is particularly suitable for such an approach because the large-scale rapid delivery of genetic constructs into the semitransparent embryos is possible. This allows for developmental analysis at a high level of resolution. The zebrafish has already become a useful system for the study of vertebrate genetics and development $(1,2)$, human hereditary diseases (3), and infectious diseases (4). In zebrafish, functional studies have relied on mutant analysis and/or inhibition of gene function by the injection of antisense morpholino oligonucleotides into the one-cell stage embryo (5). While these approaches are very efficient, they are unfortunately limited to the analysis of early gene activity.

For studying later gene activity, electroporation is an attractive approach because early gene function can be bypassed. Initially, electroporation was limited to cells in culture (6); however, subsequently, electroporation conditions have been optimized, including the usage of square wave pulses of low voltage and long duration. This approach resulted in improved survival of chick retinal explants (7) and has been adapted for in vivo studies (8).

Electroporating zebrafish embryos is not new, but previous attempts have been limited to early development or adult stages. Strategies to electroporate DNA into a whole embryo or sperm to generate transgenic zebrafish have been described $(9,10)$; however, for various reasons, these methods are not widely used. Recently, DNA electroporation into the zebrafish fin has been introduced (11).

In contrast, effective in vivo electroporation of DNA into cells of the neural tube was established in chick and mouse embryos and more recently in Xenopus tadpoles (12-14). In this case, the neural cavity was used as a DNA reservoir. The success of this approach has prompted electroporation of zebrafish genes into chick embryos (15). Thus, a need to develop an efficient system of electroporation of DNA into specific regions of the late zebrafish embryo became apparent. The challenge for performing electroporation on zebrafish embryos is to ensure the successful delivery of DNA into target tissue without damaging the yolk cell (6). Using the microinjection of DNA into the brain ventricles of the zebrafish embryo and performing electroporation in a modified conductiv- ity chamber, where the position of the brain with respect to the electrode plate determines the direction of electroporation, we succeeded in expressing green fluorescent protein (GFP) in the brain with a high survival rate. This method provides another useful addition to the quickly growing list of attractive methodological approaches that make zebrafish an even better model for developmental studies.

\section{MATERIALS AND METHODS}

\section{Materials}

The plasmid was prepared using the QIAGEN Plasmid Maxi kit (Qiagen, Hilden, Germany), solubilized in water, and its concentration adjusted to $0.5 \mu \mathrm{g} / \mu \mathrm{L}$.

\section{Electroporation Chamber}

The top three quarters of a Gene Pulser ${ }^{\circledR}$ cuvette (Bio-Rad Laboratories, Hercules, CA, USA) (Figure 1A) with a $0.4-\mathrm{cm}$ electrode gap were removed to improve the manipulation of the embryo. The chamber was half-filled with $1 \%$ molten agarose in Hank's buffer $(137 \mathrm{mM} \mathrm{NaCl}, 5.4 \mathrm{mM} \mathrm{KCl}, 0.25$ $\mathrm{mM} \mathrm{Na}_{2} \mathrm{HPO}_{4}, 0.44 \mathrm{mM} \mathrm{KH} \mathrm{PO}_{4}, 1.3$ $\mathrm{mM} \mathrm{CaCl}_{2}, 1.0 \mathrm{mM} \mathrm{MgSO} 4,4.2 \mathrm{mM}$ $\mathrm{NaHCO}_{3}, \mathrm{pH}$ 7.2). To cast a well for the embryo, a blunt microinjection pipet (Figure 1B) was inserted vertically into the center of the chamber and held in place by Parafilm ${ }^{\circledR}$, which sealed the top of the chamber [tip diameter, $0.05 \mathrm{~mm}$; outer diameter (o.d.), $1 \mathrm{~mm}$; inner diameter (i.d.), $0.58 \mathrm{~mm}$; length, $100 \mathrm{~mm}$ ] (Sutter Instruments, Novato, CA, USA). After the agarose had set, the injection needle and Parafilm were removed. Troughs that were $1 \mathrm{~mm}$ wide were constructed adjacent to the electrode plates, and Hank's buffer was added to fill them (Figure 1C). The height of the well is important; for efficient electroporation into the brain, the head must protrude from the well.

\section{Microinjection of DNA into the Neu- ral Tube of Zebrafish Embryos}

Wild-type zebrafish embryos at 24 48 hours post-fertilization (hpf) were 
grown in Hank's buffer (full-strength) containing $0.2 \mathrm{mM}$ 1-phenyl-2-thiourea (PTU) (Sigma, St. Louis, MO, USA) to inhibit pigment formation (16). Before microinjection, zebrafish embryos were anesthetized with tricaine (Sigma) and placed dorsal-side up in wedge-shape wells made in $1 \%$ agarose and covered by Hank's buffer. For injection, we used a pipet (tip diameter, $0.02 \mathrm{~mm}$ ) pulled from a glass microcapillary (o.d., $1 \mathrm{~mm}$; i.d., 0.58 $\mathrm{mm}$; length, $100 \mathrm{~mm}$ ) (Sutter Instruments). Using the micromanipulator, the pipet was inserted as described in the Figure 1D legend. DNA was injected using the MPPI-2 pressure injection system (Applied Scientific Instrumentation, Eugene, OR, USA) until the neural tube was visibly distended. The injection volume depended on the age of the embryo (Table 1). Each injected embryo was immediately transferred to the electroporation chamber.

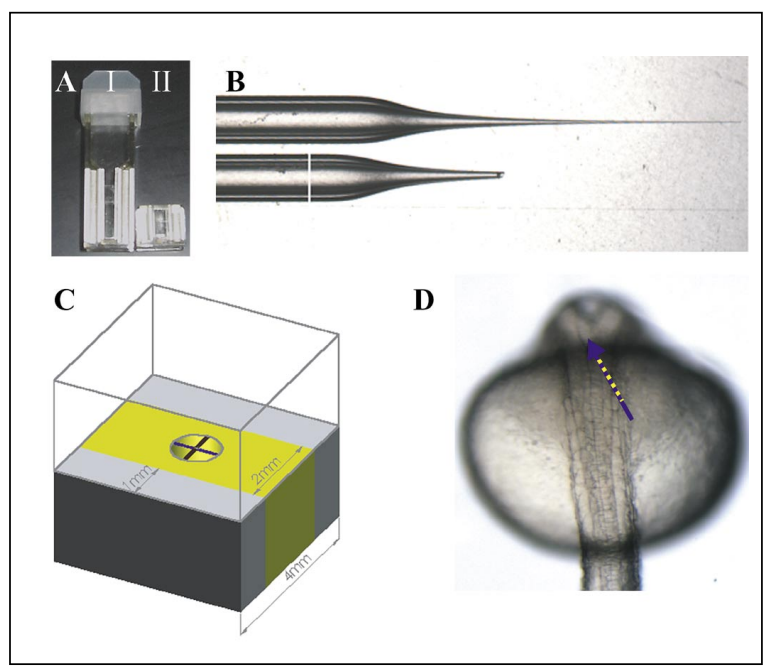

Figure 1. Equipment used for in vivo electroporation into zebrafish brain. (A) The conductivity chamber (II), formed by removing the top three quarters of a Gene Pulser cuvette (I). (B) The sharp microinjection pipet (top) and the blunt micropipet (bottom) as a mould to make the agarose well. The back of the micropipet was used to make 1-mm wide troughs that lined the sides of the electroporation chamber. (C) A diagram of the electroporation chamber. The electrode plate is shown in black, and the troughs filled with Hank's buffer are in gray. The embryo was placed in the agarose well (gray circle) surrounded by Hank's buffered agarose (yellow). The orientations of the embryo that resulted in unilateral transgene expression (blue line) or bilateral expression in the cerebellum and telencephalon (brown line) are indicated. (D) The introduction of DNA into the cavity of the neural tube. Anesthetized wild-type zebrafish embryos ( $24 \mathrm{~h}$ post-fertilization) were placed in wedgeshape wells, dorsal-side up. The pipet was pushed into the yolk cell of the embryo flanking the first somite (solid line), through the floor of the hindbrain near the otic vesicle (broken line), and into the IV ventricle (arrowhead).

\section{Electroporation}

Excess Hank's buffer was removed until it barely covered the head. The electroporation chamber was clamped generate square electric pulses (Figure 1F), phased $1 \mathrm{~s}$ apart. The conditions of electroporation are age-dependent (o.d. $\times$ height) soda-lime glass Petri dishes (Schott Glaswerke, Mainz, Germany) containing $2.5 \mathrm{~mL}$ of Hank's

\section{Fluorescence Microscopy}

The initial examination of the embryos was performed using the Olympus SZX12 stereomicroscope (Olympus, Tokyo, Japan) equipped with the ultraviolet lamp and GFP filter. For a detailed examination, the embryos expressing GFP were anesthetized in tricaine, mounted in $0.5 \%$ low melting agarose (CAMBREX, Rockland, ME, USA), and examined using the Axioplan 2 upright epifluorescence microscope (Carl Zeiss Light Microscopy, Göttingen, Germany) with a GFP filter. Images were taken with an AxioCam HRC digital camera (Carl Zeiss Vision $\mathrm{GmbH}$, Hallbergmoos, Germany). High-resolution images were taken with an LSM510 confocal laser-scanning microscope (Carl Zeiss Vision $\mathrm{GmbH})$.

\section{Whole-Mount In Situ Hybridization}

Embryos were processed for whole-mount in situ hybridization (17) using a digoxigenin-labeled (Roche Applied 
Table 1. The Effect of Pulse Conditions on Survival and Enhanced Green Fluorescent Protein (EGFP) Expression in Electroporated Zebrafish Embryos

\begin{tabular}{|c|c|c|c|c|c|c|c|c|c|}
\hline \multirow{2}{*}{$\begin{array}{l}\text { Stage of } \\
\text { Development } \\
\text { (hpf) }\end{array}$} & \multirow{2}{*}{$\begin{array}{l}\text { Pulse } \\
\text { Conditions }\end{array}$} & \multicolumn{3}{|c|}{$\begin{array}{l}\text { Neuronal and } \\
\text { Skin Expression }\end{array}$} & \multirow{2}{*}{$\begin{array}{c}\text { Total } \\
\text { Neuronal }\end{array}$} & \multirow{2}{*}{$\begin{array}{l}\text { Skin } \\
\text { Only }\end{array}$} & \multirow{2}{*}{$\begin{array}{l}\text { Deformed } \\
\text { Embryos }\end{array}$} & \multirow[b]{2}{*}{ Total } & \multirow{2}{*}{$\begin{array}{l}\text { Injection } \\
\text { Volume/nL }\end{array}$} \\
\hline & & FB/MB/HB & FB/MB & MB/HB & & & & & \\
\hline 24 & $\begin{array}{l}20 \mathrm{~V}, \\
5 \text { pulses, } \\
50 \mathrm{~ms} \text { each }\end{array}$ & 6 & 3 & 10 & 19 & 3 & 12 & 34 & $4-6$ \\
\hline 30 & $\begin{array}{l}20 \mathrm{~V}, \\
5 \text { pulses, } \\
50 \mathrm{~ms} \text { each }\end{array}$ & 1 & 8 & 2 & 11 & 18 & 2 & 31 & $4-6$ \\
\hline 36 & $\begin{array}{l}25 \mathrm{~V}, \\
5 \text { pulses, } \\
50 \mathrm{~ms} \text { each }\end{array}$ & 2 & 3 & 0 & 5 & 2 & 23 & 30 & $5-7$ \\
\hline 42 & $\begin{array}{l}25 \mathrm{~V}, \\
5 \text { pulses, } \\
50 \mathrm{~ms} \text { each }\end{array}$ & 0 & 5 & 0 & 5 & 9 & 16 & 30 & $8-10$ \\
\hline 48 & $\begin{array}{l}25 \mathrm{~V}, \\
5 \text { pulses, } \\
50 \mathrm{~ms} \text { each }\end{array}$ & 2 & 13 & 0 & 15 & 5 & 10 & 30 & $8-10$ \\
\hline
\end{tabular}

Science, Mannheim, Germany) antisense GFP RNA probe. For cryosectioning, the embryos were embedded in molten Bacto ${ }^{\mathrm{TM}}$ agar (Difco, Detroit, MI, USA) and sectioned (18).

\section{RESULTS AND DISCUSSION}

\section{Optimization of Electroporation}

Embryos (24 hpf) were used to optimize conditions for in vivo electroporation into the brain. To analyze transfection efficiency, we used pEGFPN2, a plasmid carrying the GFP gene downstream of the ubiquitous cytomegalovirus (CMV) promoter. By this stage, the neural tube is hollow and contains wider ventricles in the brain and a narrower central canal in the spinal cord. Therefore, after a single injection of DNA, which fills these cavities, the reporter vector could be electroporated anywhere in the brain. To determine the electroporation efficiency and embryo viability, embryos between 24-48 hpf were exposed to varying electroporation conditions (Table 1). Successful electroporation into the brain was observed only when square pulses of 20 volts (V) or more were used. Lower voltage caused GFP expression in the skin only (data not shown). Maximal GFP expression in the nervous tissue was observed when 24 hpf embryos were electroporated. In this case, more than half of the embryos displayed intense expression (Table 1). To electroporate 36-48 hpf embryos, the voltage was increased from 20 to $25 \mathrm{~V}$. In these embryos, expression was more localized (data not shown).

\section{GFP Expression After Electroporation}

GFP signals were detected in the skin $2 \mathrm{~h}$ after electroporation, while
GFP expression in the brain appeared after $4 \mathrm{~h}$ (data not shown). Maximal GFP expression was detected $12 \mathrm{hpf}$ in the forebrain to hindbrain (Figure 2A), midbrain to hindbrain (Figure $2 \mathrm{~B}$ ), and forebrain to midbrain (Figure 2, C-F). GFP was expressed in a unilateral manner in all embryos (Figure 2, F-J). The telencephalon was not targeted due to electroporation along the medial-lateral axis of the neural tube. GFP expression in the telencephalon can be seen when electroporation is applied along the anterior-posterior axis ( $22 \mathrm{~V}, 5$ pulses, $55 \mathrm{~ms} /$ pulse) such that the telencephalon faced the anode. Telencephalic expression is often accompanied by intense bilateral GFP expression in the cerebellum. Overall, 18 embryos of 20 survived this operation. Seventy-seven percent of the embryos (14 of 18) expressed GFP in the telencephalon, and close to $40 \%$ (7 of 18) expressed GFP in the cerebellum. However, no expres- 

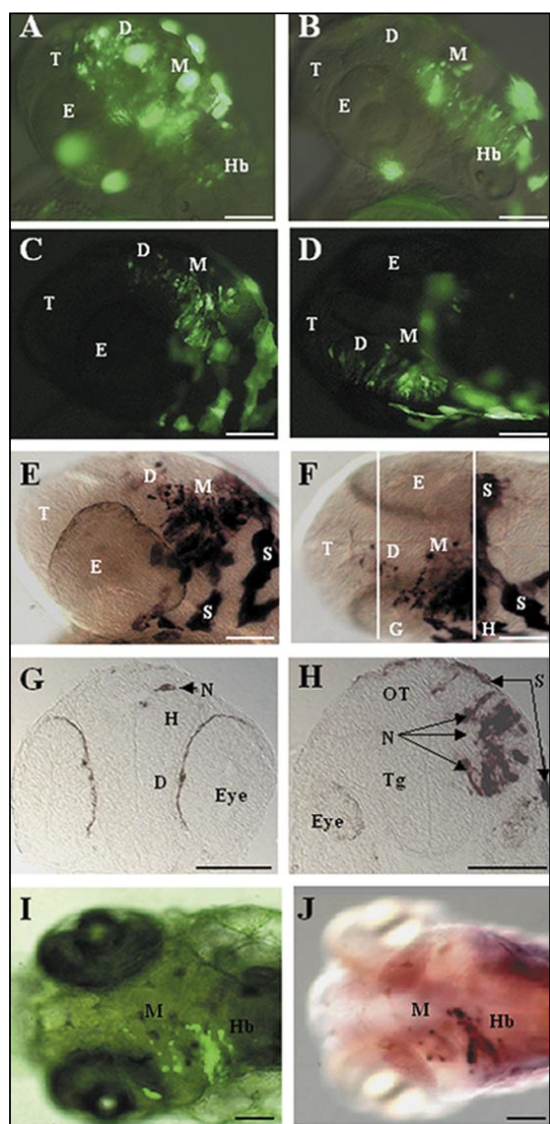

Figure 2. The range of green fluorescent protein (GFP) expression in electroporated embryos. (A and B) GFP was expressed in (A) the forebrain to hindbrain or (B) the midbrain and hindbrain 12 $\mathrm{h}$ after the electroporation of embryos that were $24 \mathrm{~h}$ post-fertilization. The merged bright-field and fluorescence photographs present embryos in side view with anterior to the left. (C and D) GFP expression in the forebrain and midbrain. The embryo is shown in side view (C) with the anterior to the left and (D) dorsal view. The majority of GFP expression was observed on the left side of the embryo that was adjacent to the anode during electroporation. ( $\mathrm{E}$ and $\mathrm{F}$ ) The embryo shown in panels $\mathrm{C}$ and $\mathrm{D}$ was stained by whole-mount in situ hybridization using anti-gfp RNA probe and presented in the same orientation. The expression pattern of $g f p$ transcripts is the same as that of GFP protein detected by fluorescence microscopy. The white bars show the positions of cross sections shown in panels $\mathrm{G}$ and $\mathrm{H}$. ( $\mathrm{G}$ and $\mathrm{H}$ ) Cross sections of the embryo shown in panels C-F at the level of $(\mathrm{G})$ the forebrain and $(\mathrm{H})$ the midbrain demonstrated the expression of $g f p$ in the neurons and skin. (I and J) The electroporation of GFP does not affect the development of the embryo. GFP expression in the brain can be detected 10 days after electroporation. (I) The merged bright-field and fluorescence photograph present an embryo in dorsal view with anterior to the left. The same embryo was processed for whole-mount in situ hybridization using $(\mathrm{J})$ anti- $g f p$ RNA probe. Scale bar $=100$ $\mu \mathrm{m}$. Abbreviations: D, diencephalons; E, eye; $\mathrm{H}$, habenula; Hb, hindbrain; M, midbrain; $\mathrm{N}$, neuron; OT, optic tectum; S, skin; T, telencephalon; Tg, tegmentum. sion was seen in the anterior spinal cord when the embryo was placed in the opposite orientation, with the hindbrain facing the anode. Thus, our conditions favor electroporation into the brain.

Whole-mount in situ hybridization using a digoxigenin-labeled anti-GFP riboprobe (Figure 2, E and $\mathrm{F}$ ) recapitulated the pattern of GFP expression detected by fluorescent microscopy (Figure 2, C and D). The same embryo was sectioned, and $g f p$ transcripts were detected in the dorsal diencephalon, including the left habenula (Figure 2G), and more posteriorly in the optic tectum and tegmentum of the midbrain (Figure $2 \mathrm{H}$ ). This means that the midbrain was electroporated at all levels along the dorsoventral axis. In addition, the
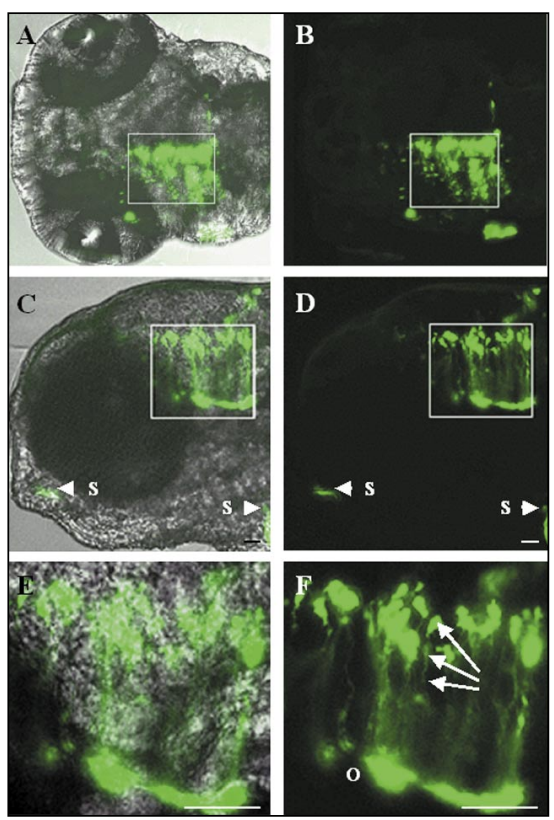

Figure 3. Confocal imaging of zebrafish embryo 4 days post-electroporation. (A and $\mathrm{B}$ ) One-sided green fluorescent protein (GFP) expression in the brain 4 days post-electroporation. The dorsal view with anterior to the left. GFP expression was confined to the left side of the brain. (A) Merged bright-field and fluorescence and (B) the corresponding fluorescent image. $(\mathrm{C}$ and $\mathrm{D})$ The same embryo in lateral view with anterior to the left. GFP is expressed in the midbrain and hindbrain. (E) Merged bright-field image and fluorescent image and (F) the corresponding fluorescent image. ( $\mathrm{E}$ and $\mathrm{F}$ ) The enlarged view of the boxed regions in panels $\mathrm{C}$ and $\mathrm{D}$, respectively. (E) Merged bright-field image and fluorescent image and (F) the corresponding fluorescent image. Arrows indicate a cell body in the ventricular zone with long processes. Scale bar: $50 \mu \mathrm{m}$. Abbreviations: O, outer region of the neural tube; $\mathrm{S}$, skin. 
cells of the skin were also $g f p$-positive (Figure 2H). These cells demonstrated a typical morphology of epithelial cells. In general, the fluorescence signal in the neural tube started to decline 3 days after electroporation. However, in a small subset of embryos, GFP fluorescence remained visible 10 days after electroporation (Figure 2, I and J).

The morphology of embryos expressing GFP was examined under the confocal microscope 4 days post-electroporation (Figure 3). In this sample, the left side of the brain was targeted, and GFP-positive cells extended from the midbrain to the hindbrain (Figure 3, A-D). GFP-positive cells were detected in the medial and lateral region of the dorsal hindbrain (Figure 3F). We noticed that some of these cells had long processes (Figure 3F, arrows). This region of the brain contains premigratory neuroblasts and radial glial cells (RGCs). RGCs are characterized by long processes directed ventrally (19). Therefore, in this case, the GFPpositive cells probably represent neuroblasts and RGCs. A second region of GFP expression was found ventrally in the region known to contain postmigratory differentiating neurons (18). Therefore, expression of GFP was detected in the early and late neurons as well as RGCs. Thus, a combination of electroporation and 3-D in vivo imaging represents a powerful approach to study the differentiation of neurons and glial cells in zebrafish development.

The modification to the in vivo electroporation method presented here is substantially different from earlier attempts to use electroporation in the zebrafish. First, a DNA solution is injected into the brain. Second, the embryo is not in direct contact with the electrode plate. Importantly, only a small part of the embryo is exposed to the electric pulse so that only cells within the exposed region are transformed. The rest of the embryo remains within the agarose well, thus minimizing the effect of the electric field on the embryo. Using this method, multiple constructs can be delivered simultaneously into the embryo. Further complexity could be achieved by the introduction of bicistronic vectors used previously for injection into one-cell stage embryos (20).
In summary, we have developed an efficient procedure for the electroporation of DNA into the brain of the zebrafish embryo. The success and reproducibility of our experiments were determined by four factors, ( $i$ ) usage of a modified electroporation chamber instead of electrodes; (ii) the optimized injection volume; (iii) the ability to rotate the embryo within the well; and (iv) electroporation that immediately followed microinjection.

\section{ACKNOWLEDGMENTS}

We thank Steven Fong, Alexander Emelyanov, and Jonathan Clarke for their helpful discussions. This project was supported by a research grant from the Agency for Science, Technology and Research (A-STAR) of Singapore (V.K.).

\section{REFERENCES}

1.Kimmel, C.B., W.W. Ballard, S.R. Kimmel, B. Ullmann, and T.F. Schilling. 1995. Stages of embryonic development of the zebrafish. Dev. Dyn. 203:253-310.

2.Haffter, P., M. Granato, M. Brand, M.C. Mullins, M. Hammerschmidt, D.A. Kane, J. Odenthal, F.J. van Eeden, et al. 1996. The identification of genes with unique and essential functions in the development of the zebrafish. Development 123:1-36.

3.Dooley, K. and L.I. Zon. 2000. Zebrafish: a model system for the study of human disease. Curr. Opin. Genet. Dev. 10:252-256.

4.Davis, J.M., H. Clay, J.L. Lewis, N. Ghori, P. Herbomel, and L. Ramakrishnan. 2002. Real-time visualization of mycobacteriummacrophage interactions leading to initiation of granuloma formation in zebrafish embryos. Immunity 17:693-702.

5.Nasevicius, A. and S.C. Ekker. 2000. Effective targeted gene "knockdown" in zebrafish. Nat. Genet. 26:216-220

6.Swartz, M., J. Eberhart, G.S. Mastick, and C.E. Krull. 2001. Sparking new frontiers: using in vivo electroporation for genetic manipulations. Dev. Biol. 233:13-21.

7.Pu, H. and A.P. Young. 1990. Glucocorticoid-inducible expression of a glutamine synthetase-CAT-encoding fusion plasmid after transfection of intact chick retinal explant cultures. Gene 89:259-263.

8.Muramatsu, T., Y. Mizutani, and J. Okumura. 1996. Live detection of the firefly luciferase gene expression by bioluminescence in incubating chick embryos. Ann. Sci. Technol. 67:906-909.

9.Buono, R.J. and P.J. Linser. 1992. Transient expression of RSVCAT in transgenic zebrafish made by electroporation. Mol. Mar. Biol. Biotechnol. 1:271-275.

10.Patil, J.G. and H.W. Khoo. 1996. Nuclear internalization of foreign DNA by zebrafish spermatozoa and its enhancement by electroporation. J. Exp. Zool. 274:121-129.

11.Tawk, M., D. Tu, Y. Torrente, S. Vriz, and D. Paulin. 2002. High-efficiency gene transfer into adult fish: a new tool to study fin regeneration. Genesis 32:27-31.

12.Nakamura, H. and J. Funahashi. 2001. Introduction of DNA into chick embryos by in ovo electroporation. Methods 24:43-48.

13.Saito, T. and N. Nakatsuji. 2001. Efficient gene transfer into the embryonic mouse brain using in vivo electroporation. Dev. Biol. 240: 237-246.

14.Haas, K., W.C. Sin, A. Javaherian, Z. Li, and H. Cline. 2001. Single-cell electroporation for gene transfer in vivo. Neuron 29: 583-591.

15.Müller, F., S. Albert, P. Blader, N. Fischer, M. Hallonet, and U. Strähle. 2000. Direct action of the nodal-related signal cyclops in induction of sonic hedgehog in the ventral midline of the CNS. Development 127:38893897.

16.Westerfield, M. 1995. The Zebrafish Book: A Guide for the Laboratory Use of Zebrafish (Brachydanio rerio). University of Oregon Press, Eugene, OR.

17.Oxtoby, E. and T. Jowett. 1993. Cloning of the zebrafish krox-20 gene (krx-20) and its expression during hindbrain development. Nucleic Acids Res. 21:1087-1095.

18.Korzh, V., T. Edlund, and S. Thor. 1993. Zebrafish primary neurons initiate expression of the LIM homeodomain protein Isl-1 at the end of gastrulation. Development 118:417-425.

19.Trevarrow, B., D.L. Marks, and C.B. Kimmel. 1990. Organization of hindbrain segments in the zebrafish embryo. Neuron 4: 669-679.

20.Wang, X., H. Wan, V. Korzh, and Z. Gong. 2000. Use of an IRES bicistronic construct to trace expression of exogenously introduced mRNA in zebrafish embryos. BioTechniques 29:814-818.

Received 10 July 2003; accepted 15 September 2003.

Address correspondence to Vladimir Korzh, Institute of Molecular and Cell Biology, 1 Research Link, Singapore, 117604. e-mail: vlad@imcb.a-star.edu.sg 